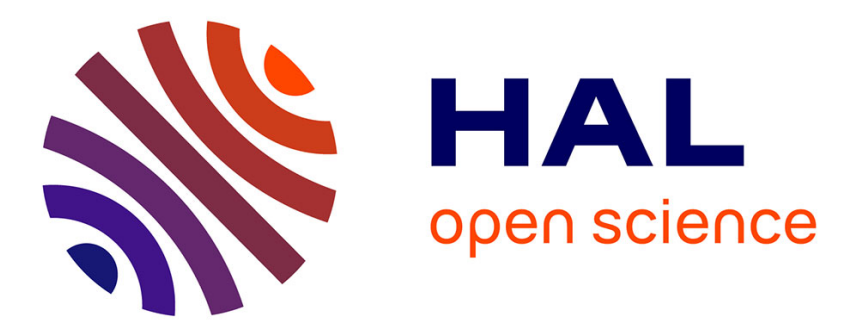

\title{
Ensuring k-coverage in low-power wide area networks for Internet of Things
}

Zhuocheng Li, Tuong-Bach Nguyen, Quentin Lampin, Isabelle Sivignon, Steeve Zozor

\section{To cite this version:}

Zhuocheng Li, Tuong-Bach Nguyen, Quentin Lampin, Isabelle Sivignon, Steeve Zozor. Ensuring kcoverage in low-power wide area networks for Internet of Things. 2016. hal-01353801v2

\section{HAL Id: hal-01353801 https://hal.science/hal-01353801v2}

Preprint submitted on 16 Aug 2016

HAL is a multi-disciplinary open access archive for the deposit and dissemination of scientific research documents, whether they are published or not. The documents may come from teaching and research institutions in France or abroad, or from public or private research centers.
L'archive ouverte pluridisciplinaire HAL, est destinée au dépôt et à la diffusion de documents scientifiques de niveau recherche, publiés ou non, émanant des établissements d'enseignement et de recherche français ou étrangers, des laboratoires publics ou privés. 


\title{
Ensuring $k$-coverage in low-power wide area networks for Internet of Things
}

\author{
Zhuocheng $\mathrm{Li}^{* \dagger}$, Tuong-Bach Nguyen ${ }^{*}$, Quentin Lampin ${ }^{\dagger}$, Isabelle Sivignon* and Steeve Zozor* \\ * Univ. Grenoble Alpes, GIPSA-Lab, F-38000 Grenoble, France \\ Emails: \{zhuocheng.li, tuong-bach.nguyen, isabelle.sivignon, steeve.zozor\}@ gipsa-lab.grenoble-inp.fr \\ $\dagger$ Orange Labs, Meylan, France \\ Emails: \{zhuocheng1.li,quentin.lampin\}@orange.com
}

\begin{abstract}
Low-Power Wide Area Networks (LPWANs) promise to enable nationwide low-power connectivity to Internet of Things (IoT) devices. While transmission ranges meet expectations, concerns on the packet delivery ratio are voiced. Receiverside diversity is often thought as a solution to improve on delivery ratio, as well as an enabler to the geolocation of devices. In this context, we study the infrastructure density required to achieve $k$-coverage of the IoT devices, which preludes $k$-reception. To that purpose, we make use of $k$-order Voronoi diagram to compute an estimate of the gateway density in reference deployment setups: dense urban, urban and rural networks. We thus provide insights on the feasibility of $k$-coverage deployment in those scenarios.

Keywords-LPWANs, $k$-coverage, $k$-order Voronoi diagram
\end{abstract}

\section{INTRODUCTION}

Trading higher bit rates for better link budgets, LPWANs provide long range wireless connectivity to IoT devices. Such networks provide a promising alternative to traditional cellular or multi-hop networks and are indeed envisioned to provide nationwide connectivity over industrial, scientific and medical (ISM) bands to battery-powered IoT devices that transmit little amount of data over long periods of time, e.g., water \& gas meters. As stated in European Telecommunications Standards Institute (ETSI) requirements for smart city applications [1], typical use cases exhibit stringent Quality of Service (QoS) requirements, e.g., high packet delivery ratio, which pose a challenge to LPWANs such as [2, 3] that mostly rely on random, uncoordinated access schemes. Those requirements, as well as harsh propagation environments, e.g., meters deployed in water pits, motivate the use of redundancy mechanisms at the emitter side, i.e., retransmissions and the receiver side, i.e., multiple-reception or receiver diversity. Unlike retransmission mechanisms that apply a higher pressure on the medium usage, receiver diversity comes at virtually no cost for IoT devices and medium usage. However, receiver diversity requires a higher infrastructure density and thus higher deployment costs.

In this context, we investigate the dimensioning of the gateway density to ensure $k$-coverage, i.e., $k$ gateways in communication range of each IoT device. The number of IoT devices being huge, we consider $k$-covering the network region of IoT devices. To this purpose, we introduce some notions based on $k$-order Voronoi diagram to express the inter-gateway distance and gateway density in three regular deployment patterns: triangular, square and hexagonal as a function of the target coverage degree $k$ and communication range $r$.
Our results provide an estimate of the infrastructure density required in three real-world reference IoT scenarios : a dense urban setup, an urban setup and a rural setup, by considering only the factor of $k$-coverage. As we will see, the $k$-coverage is not the primary dimensioning factor in some use cases. Capacity of gateways should also be taken into account.

The paper is organized as follows. In section II, we provide an overview of methodologies and results of related works in the domain of wireless sensor networks (WSNs). In section III, we introduce our problem setting and essential definitions before proposing our methodology in section IV. Section $\mathrm{V}$ is finally dedicated to the numerical application of our methodology on three network dimensioning use cases.

\section{RELATED WORKS}

$k$-order Voronoi diagram and $k$-coverage in WSNs are both well-studied subjects in the geometry and the network litteratures respectively. Without pretending to be exhaustive on the matter, we present works whose methodologies are similar to that of this paper. WSNs have been first thought to be a viable solution to monitor physical events, be it for military or civil surveillance applications. From this context arose the concept of sensing coverage, i.e., ensuring the detection of events in a monitored area. The requirement of $k$-coverage comes from the detection precision concern. In [4-7], a dense network of randomly scattered sensors is assumed and the authors propose algorithms, either distributed or centralized, to compute minimal sets of active nodes to ensure a complete $k$ coverage of the area of interest while maximizing the network lifetime. While the coverage objective is similar to covering an area of sensor nodes with gateways, such algorithms are ill-suited to compute the minimal spatial density of an infrastructure tailored to ensure $k$-coverage.

Pattern-based deployments have the advantage of guaranteeing regular inter-site distances [8]. It's especially adapted for planned infrastructure deployment. In [9], by combining the notion of grid coverage and that of $k$-perimeter coverage, sufficient yet not necessary conditions for $k$-coverage in regular deployment patterns are given. In this article, we propose a different methodology based on $k$-order Voronoi diagram that gives the necessary and sufficient condition for $k$ coverage, thus resulting in minimal spatial densities of regular deployments. Our results confirm those obtained in $[10,11]$ in 
terms of optimal 1 and 2-coverage deployment patterns, and can give some insights for the more general case of $k \geq 3$.

$k$-order Voronoi diagram has been used to study distributed and randomized $k$-coverage issues in WSNs $[12,13]$. However, pattern-based deployments being more appropriate for infrastructure deployment by requiring probably smaller deployment density, we apply $k$-order Voronoi diagram to regular patterns and find the optimal $k$-coverage pattern as well as an overview of the deployment cost for achieving $k$-coverage.

\section{PRoblem Formulation}

As in [9], in order to model the gateway deployment, we choose three regular patterns coming from the only three possible edge-to-edge tilings of the $2 \mathrm{D}$ plane by mutually congruent regular square, triangle and hexagon polygons [8]. The vertices of the polyons represent the positions of gateways, as shown in figure 1. As the deployments thus obtained are spatially periodical, single periods are shown. We first introduce several definitions necessary to our problem formulation.

We denote the Euclidean distance between two points $p, q \in \mathbb{R}^{2}$ by $d(p, q)$. The region to provide $k$-coverage is denoted by $\Omega \subseteq \mathbb{R}^{2}$, that can be bounded or not. Let $G$ denote the deployment pattern of gateways, $G=\left\{g_{i}\right\}_{i \in I}$, where $I \subseteq \mathbb{N}$. $G$ is not necessarily limited to $\Omega$, nor finite (assuming that the pattern is regular, when $\Omega=\mathbb{R}^{2}$ the pattern is necessarily infinite). Let $|\cdot|$ denotes the cardinality, and $r$ the communication range which is supposed to be identical for all devices. We denote by $G(p, r)$ the subset of $G$ enclosed by a disc of radius $r$, centered at $p \in \Omega$, i.e., $G(p, r)=\left\{g_{i} \in G \mid d\left(p, g_{i}\right) \leq r\right\}$. Finally, the $k$-distance of $p$ to set $G$ is defined as $d_{k}(p, G)=\min \{r \geq 0|| G(p, r) \mid \geq k\}$.

$k$-coverage means that every device in $\Omega$ is able to communicate with $k$ gateways in $G$ with communication range $r$ as stated by the following definition and proposition.

Definition 1 ( $k$-coverage). $\Omega$ is said to be $k$-covered by the deployment $G$, if for any $p \in \Omega$ representing a device, $|G(p, r)| \geq k$.

Proposition 1. The minimal communication range to $k$-cover $\Omega$ is $r_{k}=\max _{p \in \Omega} d_{k}(p, G)$.

Proof. Note that since $G$ is defined as a regular pattern, this maximum is well defined as long as $k \leq|G|$. If $r_{k}$ is the minimal communication range achieving $k$-coverage, then we have $r_{k} \geq \max _{p \in \Omega} d_{k}(p, G)$ : indeed, if $r_{k}<\max _{p \in \Omega} d_{k}(p, G)$, there exists at least a point $p$ such that $|G(p, r)|<k$ and $p$ is not $k$ covered by definition. For $r=\max _{p \in \Omega} d_{k}(p, G)$, we have for any $p \in \Omega, r \geq d_{k}(p, G)$, according to the definition of $d_{k}(p, G)$, we have $|G(p, r)| \geq k$, thus $\Omega$ is $k$-covered.

In order to find $r_{k}$, let's introduce the following definitions.

Definition 2 ( $k$-order Voronoi cell). Let us denote by $\mathscr{P}_{k}(G)=\{\mathscr{G} \subseteq G|| \mathscr{G} \mid=k\}$ the collection of the subsets of $G$ having the cardinality $k$, with $k \geq 1$. For any $\mathscr{G}_{0} \in \mathscr{P}_{k}(G)$, the $k$-order Voronoi cell of $\mathscr{G}_{0}$ is defined as $C_{k}\left(\mathscr{G}_{0}\right)=\left\{p \in \Omega \mid \forall \mathscr{G} \in \mathscr{P}_{k}(G), d_{k}\left(p, \mathscr{G}_{0}\right) \leq d_{k}(p, \mathscr{G})\right\}$.
Definition 3 ( $k$-order Voronoi cell of a vertex). The $k$-order Voronoi cell of a vertex $g_{i} \in G$ is defined as $V_{k}\left(g_{i}\right)=$ $\bigcup_{\mathscr{G} \ni g_{i}} C_{k}(\mathscr{G})$.

$C_{k}\left(\mathscr{G}_{0}\right)$ is the set of points that have $\mathscr{G}_{0}$ as the $k$ nearest gateways among $G$. $V_{k}\left(g_{i}\right)$ is the set of points that have $g_{i}$ as one of its $k$ nearest gateways among $G$. Voronoi polygon of $g_{i}$ is a special $k$-order Voronoi cell given by $k=1$, i.e., $C_{1}\left(g_{i}\right)=V_{1}\left(g_{i}\right)$. Note that there is no overlap between adjacent $C_{k}$, but $V_{k}$ overlap in general except for $V_{1}$. An example of $C_{3}(\mathscr{G}), V_{3}\left(g_{i}\right)$ and $C_{1}\left(g_{i}\right)=V_{1}\left(g_{i}\right)$ is shown in figure 1 . With all the necessary definitions given, in section IV we express the minimal $k$-coverage ensuring density $D(k, r)$ in regular patterns by fixing a communication range $r$.

\section{ENSURING $k$-COVERAGE}

We define the spatial density of a deployment as the number of gatways per unit surface, i.e., $D=\frac{|G|}{S_{\Omega}}$, where $S_{\Omega}$ stands for the surface of $\Omega$. When $\Omega$ and $G$ are infinite, $D=\frac{1}{S_{\mathrm{vp}}}$ where $S_{\mathrm{vp}}$ is the surface of the Voronoi polygon $V_{1}\left(g_{i}\right)$ of the regular pattern. In this section, we first consider the infinite case $\Omega=\mathbb{R}^{2}$. A realistic finite scenario with $\Omega$ chosen as a rectangle region is briefly discussed at the end of the section.

\section{A. Necessary and sufficient condition of $k$-coverage}

Proposition 2. We denote the inter-gateway distance in regular patterns by a, i.e., the distance between nearest gateways. By fixing an a, the necessary and sufficient condition for $\mathbb{R}^{2}$ to be $k$-covered is that every point $g_{i}$ in $G$ covers $V_{k}\left(g_{i}\right)$. $r_{k}$ equals to the distance between an arbitrary vertex $g_{i}$ and the most distant points from $g_{i}$ in $V_{k}\left(g_{i}\right)$. We have $r_{k}(a)=\max _{p \in \mathbb{R}^{2}} d_{k}(p, G)=\max _{p \in V_{k}\left(g_{i}\right)} d\left(p, g_{i}\right)$.

Proof. Since $C_{k}\left(\mathscr{G}_{0}\right)$ perfectly tessellate the 2D plane, i.e., $\bigcup C_{k}\left(\mathscr{G}_{0}\right)=\mathbb{R}^{2}$, we have $r_{k}(a)=$ $\mathscr{G}_{0} \in \mathscr{P}_{k}(G)$

$\max \max _{k} d_{k}(p, G)$. According to the definition $\mathscr{G}_{0} \in \mathscr{P}_{k}(G) p \in C_{k}\left(\mathscr{G}_{0}\right)$
of $d_{k}(p, G)$ and $C_{k}\left(\mathscr{G}_{0}\right)$, we have for any $p \in$ $C_{k}\left(\mathscr{G}_{0}\right), d_{k}(p, G)=\min _{\mathscr{G} \in \mathscr{P}_{k}(G)} d_{k}(p, \mathscr{G})=d_{k}\left(p, \mathscr{G}_{0}\right)=$ $\max _{g_{i} \in \mathscr{G}_{0}} d\left(p, g_{i}\right)$. Thus $r_{k}(a)=\max _{\mathscr{G}_{0} \in \mathscr{P}_{k}(G)} \max _{p \in C_{k}\left(\mathscr{G}_{0}\right)} \max _{g_{i} \in \mathscr{G}_{0}} d\left(p, g_{i}\right)$. $g_{i} \in \mathscr{G}_{0}$
Let's change the order of $\max$ to have $r_{k}(a)=$ $\max \max \max d\left(p, g_{i}\right)$, which can be rewritten $g_{i} \in G \mathscr{G}_{0} \in \mathscr{P}_{k}(G), \mathscr{G}_{0} \ni g_{i} p \in C_{k}\left(\mathscr{G}_{0}\right)$

as $r_{k}(a)=\max _{g_{i} \in G} \max _{p \in V_{k}\left(g_{i}\right)} d\left(p, g_{i}\right)$ according to the definition of $V_{k}\left(g_{i}\right)$. Recall that $G$ is regular pattern, naturally $V_{k}\left(g_{i}\right)$ takes the same form, we can thus remove the $\max$ on $g_{i}$, which gives us $r_{k}(a)=\max _{p \in V_{k}\left(g_{i}\right)} d\left(p, g_{i}\right)$.

The proof of propostion 2 is inspired from [14]. Trivially, the coverage condition is scale-invariant and thus $r_{k}(a)$ is proportional to $a$, i.e., $r_{k}(a)=\beta(k) a$ where $\beta(k)$ depends only on $k$ and on the pattern.

Proposition 3. Given a communication range $r$, the minimal densities $D(k, r)$ ensuring $k$-coverage in regular patterns is 
given by $D(k, r)=\frac{1}{S_{\mathrm{vp}}\left(a_{k}(r)\right)}$, where $a_{k}(r)=\frac{r}{\beta(k)}$ is the maximal inter-gateway distance that guarantees $k$-coverage.

Proof. If $a=a_{k}(r)=\frac{r}{\beta(k)}$, we have $r=\beta(k) a=r_{k}(a)$, according to proposition 2 , we guarantee $k$-coverage.

We illustrate an example of 3-coverage by proposition 3 in figure 1. Obviously, $D(k, r)$ is proportional to $\frac{1}{a_{k}(r)^{2}}$, and thus to $\frac{1}{r^{2}}$, i.e., $D(k, r)=\frac{\alpha(k)}{r^{2}} . \alpha(k)$ is the $k$-coverage coefficient which depends only on $k$ and on the pattern. $D(k, r)$ can be expressed as: $D_{\mathrm{sqr}}(k, r)=\frac{1}{a_{k}(r)^{2}}$, so that $\alpha_{\mathrm{sqr}}(k)=\beta_{\mathrm{sqr}}(k)^{2}$ for the square pattern; $D_{\operatorname{tri}}(k, r)=\frac{2 \sqrt{3}}{3 a_{k}(r)^{2}}$, so that $\alpha_{\text {tri }}(k)=$ $\frac{2 \sqrt{3} \beta_{\text {tri }}(k)^{2}}{3}$ for the triangular pattern; $D_{\text {hex }}(k, r)=\frac{4 \sqrt{3}}{9 a_{k}(r)^{2}}$, so that $\alpha_{\text {hex }}(k)=\frac{4 \sqrt{3} \beta_{\text {hex }}(k)^{2}}{9}$ for the hexagonal pattern. $D(k, r)$ is thus directly linked to $\beta(k)$ which can be determined exactly by some algorithm of the literatures on $k$-order Voronoi diagrams (see for instance [16]).
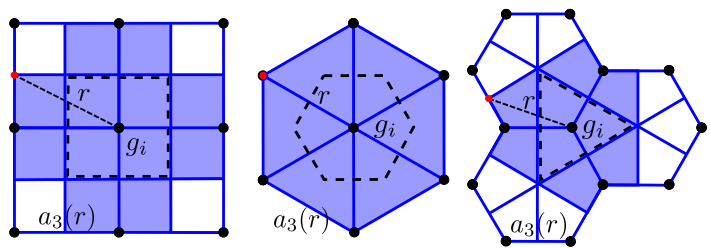

Fig. 1. Illustration of three regular patterns based deployments. Black points are the positions of gateways. Polygons with blue edges are $C_{3}(\mathscr{G})$ Regions painted in light blue are $V_{3}\left(g_{i}\right)$, i.e., $\cup_{\mathscr{G} \ni g_{i}} C_{3}(\mathscr{G})$. Polygons with dashed black edges are $C_{1}\left(g_{i}\right)=V_{1}\left(g_{i}\right)$. Red points are $p=$ $\operatorname{argmax}_{p \in V_{3}\left(g_{i}\right)} d\left(p, g_{i}\right) . r$ denotes a given communication range. $a_{3}(r)$ is maximal inter-gateway distance for 3-coverage with the given $r$.

\section{B. Numerical results}

$\alpha(k)$ for three regular patterns are listed in table $\mathrm{I}$. We observe that $\alpha_{\text {tri }}(1) \approx 0.385$ is the smallest for 3 regular patterns, which confirms the theory of [10], i.e., the regular triangular pattern is asymptotically optimal in terms of the number of discs necessary to provide 1-coverage on a plane. We define the minimal $k$-coverage achieving coefficient in mutually congruent patterns as $\alpha_{\min }$. Obviously, we have $\alpha_{\min }(1)=\alpha_{\operatorname{tri}}(1)$. In [11], the authors find that $\alpha_{\min }(2)=\frac{4}{3 \sqrt{3}}$, which coincides with $2 \alpha_{\text {tri }}(1)$, meaning a superposition of two 1-coverage regular triangular patterns gives $\alpha_{\min }(2)$. Note that regular hexagonal pattern can be seen as a superposition of two regular triangular patterns, that's why we have $\alpha_{\text {hex }}(2)=2 \alpha_{\text {tri }}(1)=\alpha_{\min }(2)$. The advantage of regular hexagonal pattern over any two randomly superposed regular triangular patterns is the inter-gateway distance can not be arbitrarily small. Since $\alpha_{\min }(1)$ and $\alpha_{\min }(2)$ are found among regular patterns, we conjecture that for $k \geq 3$, $\alpha_{\min }(k)=\min \left\{\alpha_{\text {sqr }}(k), \alpha_{\text {tri }}(k), \alpha_{\text {hex }}(k)\right\}$.

In literatures, many heuristics for optimal general $k$ coverage have been proposed by superposing $k$ regular triangular patterns [17, 18]. Superposed patterns may be optimal for $k=3$, as $3 \alpha_{\text {tri }}(1)=\min \left\{\alpha_{\text {sqr }}(3), \alpha_{\text {tri }}(3), \alpha_{\text {hex }}(3)\right\}=$
TABLE I

$\alpha(k)$ IN FUNCTION OF $k$ FOR THREE REGULAR PATTERNS

\begin{tabular}{|l|c|c|c|c|c|c|}
\hline & $k=1$ & $k=2$ & $k=3$ & $k=4$ & $k=5$ & $k=6$ \\
\hline$\alpha_{\mathrm{sqr}}(k)$ & $\frac{1}{2}$ & 1 & $\frac{5}{4}$ & $\frac{25}{18}$ & $\frac{5}{2}$ & $\frac{5}{2}$ \\
\hline$\alpha_{\mathrm{tri}}(k)$ & $\frac{2 \sqrt{3}}{9}$ & $\frac{2 \sqrt{3}}{3}$ & $\frac{2 \sqrt{3}}{3}$ & $\frac{8 \sqrt{3}}{9}$ & $\frac{7 \sqrt{3}}{6}$ & $\frac{98 \sqrt{3}}{81}$ \\
\hline$\alpha_{\text {hex }}(k)$ & $\frac{4 \sqrt{3}}{9}$ & $\frac{4 \sqrt{3}}{9}$ & $\frac{7 \sqrt{3}}{9}$ & $\frac{196 \sqrt{3}}{225}$ & $\frac{4 \sqrt{3}}{3}$ & $\frac{4 \sqrt{3}}{3}$ \\
\hline
\end{tabular}

$\alpha_{\text {tri }}(3)$. Note that a 3-coverage regular triangular pattern can be decomposed to three 1-coverage regular triangular patterns. For $k=4,4 \alpha_{\text {tri }}(1) \approx 1.540>$ $\min \left\{\alpha_{\mathrm{sqr}}(4), \alpha_{\text {tri }}(4), \alpha_{\text {hex }}(4)\right\}=\alpha_{\text {sqr }}(4) \approx 1.389$. For $k=$ $5,5 \alpha_{\text {tri }}(1) \approx 1.925<\min \left\{\alpha_{\text {sqr }}(5), \alpha_{\text {tri }}(5), \alpha_{\text {hex }}(5)\right\}=$ $\alpha_{\text {tri }}(5) \approx 2.021$. For $k=6,6 \alpha_{\text {tri }}(1) \approx 2.309>$ $\min \left\{\alpha_{\mathrm{sqr}}(6), \alpha_{\text {tri }}(6), \alpha_{\text {hex }}(6)\right\}=\alpha_{\text {tri }}(6) \approx 2.096$. For $k=$ 4,6 , superposition heuristics result in greater densities. For $k=5$, they require smaller density but the resulting patterns neither are necessarily mutually congruent nor guarantee a non arbitrarily small inter-site distance. Although it's not our primary objective to deduce optimal $k$-coverage pattern, our work can give some guidelines for this effort. In Figure 2 we can see that $\alpha(k)$ increases almost linearly with $k$, revealing the scalability of $k$-coverage deployment cost.

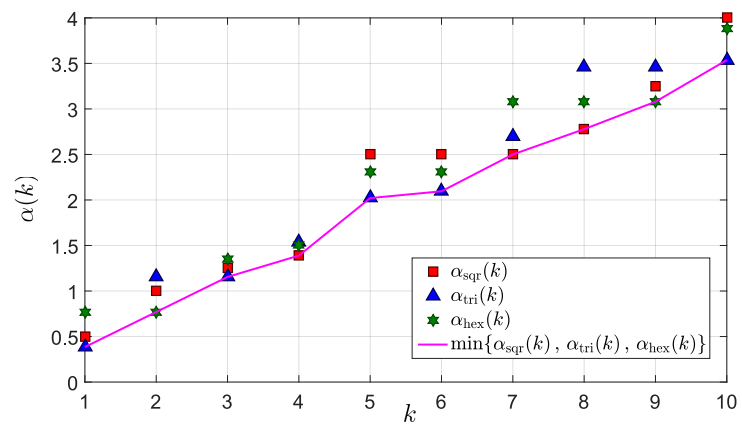

Fig. 2. $\alpha(k)$ in function of $k$ in three regular patterns

\section{Edge effect consideration}

In this section, we consider a realistic finite scenario. We first provide the following proposition.

Proposition 4. Let $\Omega \subset \mathbb{R}^{2}$ be a bounded region and $r$ a given communication range. If gateways are deployed following any regular pattern with inter-gateway distance $a_{k}(r)$ inside $\Omega^{\oplus r}=\left\{y \in \mathbb{R}^{2} \mid \exists x \in \Omega, d(x, y) \leq r\right\}$, defined as the dilation of $\Omega$ by $r$, then $\Omega$ is $k$-covered.

Proof. If we deploy gateways following any regular pattern with inter-gateway distance $a_{k}(r)$ to the whole $\mathbb{R}^{2}$, according to the proposition 3 , we have $k$-coverage over $\mathbb{R}^{2}$. As $\Omega \in \mathbb{R}^{2}$, $\Omega$ is $k$-covered. For any $g_{i} \in \mathbb{R}^{2} / \Omega^{\oplus r}$, according to the definition of $\Omega^{\oplus r}$, we have $\forall p \in \Omega, d\left(p, g_{i}\right)>r$, Recall that the coverage degree of $p$ is $|G(p, r)|$, with $G(p, r)=\left\{g_{i} \in\right.$ $\left.G \mid d\left(p, g_{i}\right) \leq r\right\}$. Removing all the $g_{i} \in \mathbb{R}^{2} / \Omega^{\oplus r}$ doesn't change $|G(p, r)|$. 


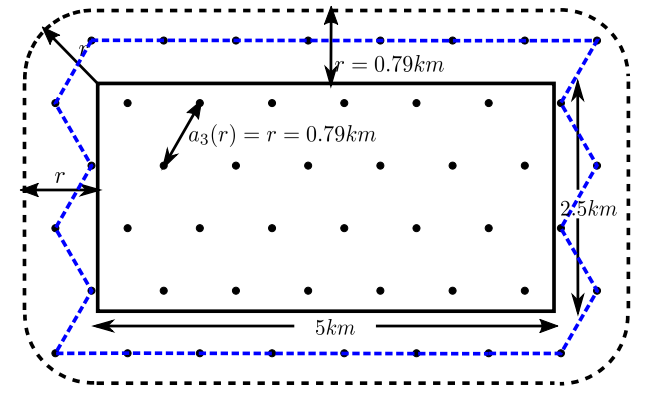

Fig. 3. Illustration of edge effect. Region enclosed by black solid line is $\Omega$, by black dashed line $\Omega^{\oplus r}$. The area between black and blue dashed line is the border area.

Let's take an example of providing 3-coverage with regular triangular pattern to a rectangle region $\Omega$ of dimensions $5 \mathrm{~km}$ $\times 2.5 \mathrm{~km} . r$ is chosen to be a realistic communication range for LPWANs in dense urban scenario, i.e., $0.79 \mathrm{~km}$, see table II. $a_{3}(r)=r=0.79 \mathrm{~km}$ for triangular pattern. One way to interpret this proposition is to consider $\Omega$ as the region of interest filled with sensor nodes to provide 3 -coverage. This proposition provides a way to fully 3 -cover $\Omega$ by deploying gateways in the dilation of it, i.e., $\Omega^{\oplus r}$. "Gateway density" in this scenario can be calculated as $D_{\text {full }}=\frac{\left|G \cap S_{\Omega} \oplus r\right|}{S_{\Omega}}=$ $\frac{48}{12.5 \mathrm{~km}^{2}}=3.84 / \mathrm{km}^{2}$, which is much greater than the density calculated in infinite case, i.e., $D_{\text {tri }}(3, r)=\frac{2 \sqrt{3}}{3 r^{2}} \approx \frac{1.155}{r^{2}} \approx$ $1.85 / \mathrm{km}^{2}$. However, if we consider $\Omega^{\oplus r}$ as the area of interest, certainly the border area of it is not 3-covered, but the center area $\Omega$ is fully 3 -covered, see figure 3 . In this scenario we have $D_{\text {realistic }}=\frac{\left|G \cap S_{\Omega \oplus r \mid}\right|}{S_{\Omega} \oplus r} \approx \frac{48}{26.31 \mathrm{~km}^{2}} \approx 1.82 / \mathrm{km}^{2}$. In the fully 3 -covered area $\Omega, D_{\text {center }}=\frac{\left|G \cap S_{\Omega}\right|}{S_{\Omega}} \approx \frac{24}{12.5 \mathrm{~km}^{2}} \approx 1.92 / \mathrm{km}^{2}$. In reality, the border area being usually less populated, it's not necessary and realistic to perfectly $k$-cover it, especially when the cost is too high compared to the realistic case. Moreover, it's difficult to consider edge effect which depends on region shapes etc. It's of little interest when providing general scaling laws. In section $\mathrm{V}$, we neglect the edge effect and stick to the approximation $D(k, r)=\frac{1}{S_{V P}\left(a_{k}(r)\right)}$ in order to evaluate the efficiency of a real LPWANs $k$-coverage deployment .

\section{APPLICATION}

In this section, by applying our results in IV, we aim at visualizing the dimensioning of a real LPWANs deployment according to the $k$-coverage criterion in some real-world scenarios. In order to quantify the efficiency of a deployment we introduce the average number of sensor nodes per gateway when $k$-coverage achieved, denoted by $N_{\text {avg }}=d_{s} / D(k, r)$, in which $d_{s}$ indicates the sensor node density in an area. Since $D(k, r)$ depends on $r$, we consider 3 scenarios, i.e., dense urban, urban and rural, in which the sensor node density $d_{s}$ and typical communication range $r$ are intrinsically different, due to different population density and radio environment.

We consider a real LPWANs technology LoRaWAN ${ }^{\mathrm{TM}}$, which operates on $868 \mathrm{MHz}$ frequency band in Europe and has a typical link budget of $154 \mathrm{~dB}$ [2]. For the path loss model, we choose Hata model for $150 \mathrm{MHz} \leq f \leq 1000 \mathrm{MHz}$ as recommended by ETSI for GSM $900 \mathrm{MHz}$ [19]. We add shadowing margin as it's not included in the Hata model and penetration loss as sensor nodes are mostly deployed indoor. Typical values of these two parameters are found in [20]. The parameters and calculated typical $r$ are listed in table II.

TABLE II

PATH LOSS PARAMETERS FOR LORAWAN ${ }^{\mathrm{TM}}$

\begin{tabular}{|l|c|c|c|}
\hline Parameters & dense urban & urban & rural \\
\hline shadowing margin $(\mathrm{dB})$ & 13 & 13 & 13 \\
\hline penetration loss $(\mathrm{dB})$ & 18 & 15 & 10 \\
\hline typical $r(\mathrm{~km})$ & 0.79 & 0.92 & 2.43 \\
\hline
\end{tabular}

$d_{s}$ can be calculated as the product of the house density and number of sensor nodes per house. For house density, we gather some statistics from INSEE [21] for different scenarios. For dense urban scenario, we take the city of Paris in 2013 as an example. For urban and rural scenarios, we average statistics from several cities and villages. The estimations of number of sensor nodes per house are also listed in table III.

TABLE III

STATISTICS AND ESTIMATIONS IN DIFFERENT SCENARIOS

\begin{tabular}{|l|c|c|c|}
\hline Statistics & dense urban & urban & rural \\
\hline house density $\left(/ \mathrm{km}^{2}\right)$ & 10946 & 2845 & 102 \\
\hline sensor nodes $/$ house & 10 & 8 & 5 \\
\hline$d_{s}\left(\right.$ nodes $\left./ \mathrm{km}^{2}\right)$ & 109460 & 22760 & 510 \\
\hline
\end{tabular}

$N_{\mathrm{avg}}=\frac{d_{s}}{D(k, r)}=d_{s} S_{\mathrm{vp}}\left(a_{k}(r)\right)$, which is the number of sensor nodes in the Voronoi polygon of a gateway $g_{i}$. These sensor nodes are nearer to $g_{i}$ than to any other gateways. However, the number of sensor nodes that can actually communicate with $g_{i}$ should be calculated as $N_{\text {real }}=d_{s} \pi r^{2} . N_{\text {avg }}$ and $N_{\text {real }}$ are both represented in figure 4. $N_{\text {avg }}$ reflects the number of sensor nodes "allocated" to $g_{i} . D(k, r)$ increases with $k, N_{\text {avg }}$ thus decreases with $k$. A denser deployment can in fact reduce the number of sensor nodes "allocated" to every gateway, thus reducing it's traffic pressure.

A gateway can only support packets from a limited number of sensor nodes, i.e., the load-capacity of gateway denoted by $C_{\text {load }}$. It depends on the number of frequency channels available, the duration of packets, the interference phenomenon between packets etc. Note that $C_{\text {load }}$ of a gateway also increases with $k$ in that packets benefit from an increase in reception diversity. One can imagine an increasing curve representing $C_{\text {load }}$ in figure 4 , which probably crosses the $N_{\text {real }}$ and $N_{\text {avg }}$ curves. As sensor nodes in LPWANs send their packets using uncoordinated access schemes, the level of interferences increases with $N_{\text {real }}$ and $N_{\text {avg. }}$. When $N_{\text {real }}$ and $N_{\text {avg }}$ are too high compared to $C_{\text {load }}$, we are in the interference-limited regime. Even with $k$-reception, there can be heavy packet loss. Therefore the position of $C_{\text {load }}$ curve is essential to decide when the dimensioning factor is the $k$ coverage or $C_{\text {load }}$. In dense scenarios, even though we deploy gateways to achieve a certain $k$-coverage, $N_{\text {real }}$ and $N_{\text {avg }}$ may be too high compared to $C_{\text {load }}$. One solution is to allocate 


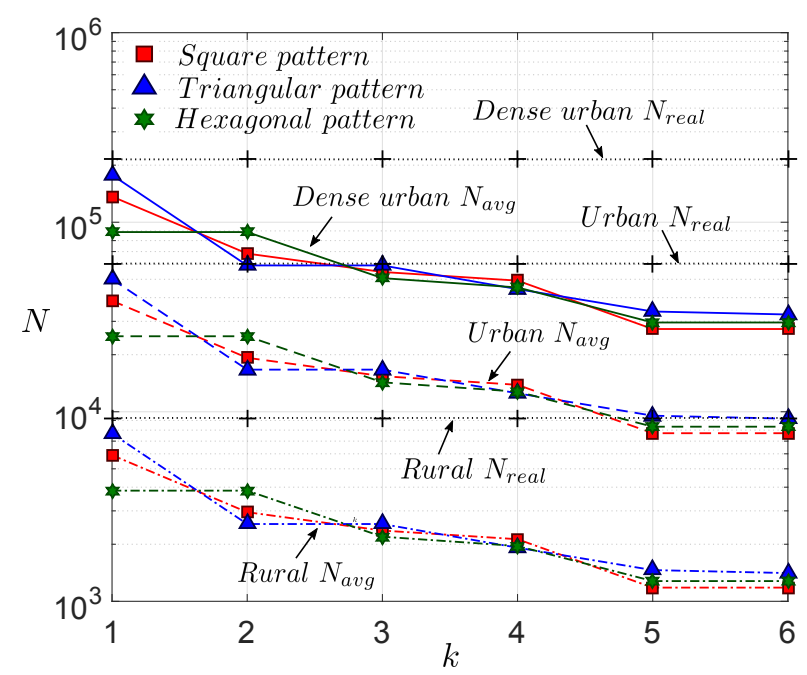

Fig. 4. Comparison of $N_{\text {avg }}$ and $N_{\text {real }}$ in 3 scenarios

more frequency resources to gateways, which is not always available. Another solution is to reduce the communication range $r$ by a tilt of the gateway antenna or an artificial reduction of the reception sensibility etc., in order to reduce $N_{\text {real }}$ and $N_{\text {avg }}$ and thus the interferences, but more gateways needs to be deployed in this case. The primary dimensioning factor is thus $C_{\text {load }}$. In sparse cases, by achieving $k$-coverage, $N_{\text {real }}$ and $N_{\text {avg }}$ don't exceed $C_{\text {load }}$ of gateways, no more gateways need to be deployed. The primary dimensioning factor is thus $k$-coverage.

In [22], the phenomenon of interferences between packets is studied using a stochastic geometry model for typical LPWANs access schemes, in order to deduce $C_{\text {load }}$ without considering $k$-reception. An extra reference $C_{\text {load }}$ curve can be drawn by combining the interference model in [22] and $k$ coverage model in this article in order to give a more refined study of the dimensioning factors of LPWANs.

\section{CONCLUSION AND FUTURE WORKS}

In this article, we study the problem of minimal gateway density ensuring $k$-coverage in regular deployment patterns in order to give some insights of the dimensioning of the LPWANs. We notice that the gateway density increases almost linearly with coverage degree, revealing that the cost of $k$ coverage is almost linear and thus scalable. Our method gives the optimal $k$-coverage patterns among the three regular ones. which confirm the results obtained in $[10,11]$. We thus conjecture that minimal density for general $k$-coverage among mutually congruent patterns is always achieved among the three regular patterns.

From three real-world scenarios, we show that $k$-coverage alone is unable to dimension the gateway density, especially in dense populated scenarios, load-capacity of gateway being another essential factor. In future works, we are going to combine the load-capacity model and $k$-coverage model in order to give a more refined dimensioning of LPWANs.

\section{REFERENCES}

[1] "ETSI." http://www.etsi .org/deliver/etsi_tr/103000_ 103099/103055/01.01.01_60/tr_103055v010101p.pdf. Accessed: 2016-08-03.

[2] "LoRaWAN ${ }^{\mathrm{TM}}$ Specification." https://www.lora-alliance. org/portals/0/documents/whitepapers/LoRaWAN101.pdf. Accessed: 2016-08-03.

[3] "Sigfox." http://www.sigfox.com/fr. 2016-08-03.

[4] S. Kumar, T. H. Lai, and J. Balogh, "On k-coverage in a mostly sleeping sensor network," in Proc. of the 10th annual int. conf. on Mobile computing and networking, pp. 144-158, 2004.

[5] G. Xing, X. Wang, Y. Zhang, C. Lu, R. Pless, and C. Gill, "Integrated coverage and connectivity configuration for energy conservation in sensor networks," ACM Trans. on Sensor Networks, vol. 1, no. 1, pp. 36-72, 2005.

[6] M. Hefeeda and M. Bagheri, "Randomized $k$-coverage algorithms for dense sensor networks," in 26th IEEE Int. Conf. on Computer Comm., pp. 2376-2380, 2007.

[7] H. M. Ammari and S. K. Das, "Centralized and clustered $k$-coverage protocols for wireless sensor networks," IEEE Trans. on Computers, vol. 61, no. 1, pp. 118-133, 2012.

[8] B. Grunbaum and G. C. Shephard, "Tilings by regular polygons," Math. Mag., vol. 50, no. 5, pp. 227-247, 1977.

[9] Y.-h. Kim, C.-M. Kim, D.-S. Yang, Y.-j. Oh, and Y.-H. Han, "Regular sensor deployment patterns for $p$-coverage and $q$-connectivity in wireless sensor networks," in Int. Conf. on Information Networking, pp. 290-295, 2012.

[10] R. Kershner, "The number of circles covering a set," Am. J. of math., vol. 61, no. 3, pp. 665-671, 1939.

[11] J. Ge, "Planar thinnest deployment pattern of congruent discs which achieves 2-coverage," School of Mathematical Sciences, Soochow University, Suzhou 215006, P. R. China, 2010.

[12] C. Zhu, C. Zheng, L. Shu, and G. Han, "A survey on coverage and connectivity issues in wireless sensor networks," Journal of Network and Computer Applications, vol. 35, no. 2, pp. 619-632, 2012.

[13] F. Li, J. Luo, S.-Q. Xin, W.-P. Wang, and Y. He, "LAACAD: Load balancing k-area coverage through autonomous deployment in wireless sensor networks," in IEEE 32nd Int. Conf. on Distributed Computing Systems, pp. 566-575, 2012.

[14] A. M.-C. So and Y. Ye, "On solving coverage problems in a wireless sensor network using voronoi diagrams," in Int. Workshop on Internet and Network Economics, pp. 584-593, 2005.

[15] D.-T. Lee et al., "On k-nearest neighbor voronoi diagrams in the plane," IEEE Trans. Computers, vol. 31, no. 6, pp. 478-487, 1982.

[16] J.-E. Kim, J. Han, and C.-G. Lee, "Optimal 3-coverage with minimum separation requirements for ubiquitous computing environments," Mobile Networks and Applications, vol. 14, no. 5, pp. 556-570, 2009. 
[17] Z. Yuan, W. Li, J. Zhu, and W. Zhao, "A cost-efficiency method on beacon nodes placement for wireless localization," in IEEE Int. Conf. on Computing, Networking and Communications, pp. 546-550, 2015.

[18] Z. Li, T.-B. Nguyen, Q. Lampin, I. Sivignon, and S. Zozor, "Ensuring k-coverage in low-power wide area networks for internet of things." https://hal.archivesouvertes.fr/hal-01353801.

[19] “ETSI." http://www.etsi .org/deliver/etsi_tr/101300 101399/101362/06.00.01_60/tr_101362v060001p.pdf. Accessed: 2016-08-03.

[20] A. R. Mishra, Advanced cellular network planning and optimisation. John Wiley \& Sons, 2007.

[21] "INSEE." http://www.insee.fr/en/themes/theme.asp? theme $=1 \&$ sous theme $=2$. Accessed: 2016-08-03.

[22] S. Zozor, Z. Li, Q. Lampin, and J.-M. Brossier, "Timefrequency aloha-like random access: A scalability study of low-power wide-area networks for iot using stochastic geometry," 